УДК $51-74$

\title{
ЧИСЛЕННОЕ РЕШЕНИЕ ОБРАТНЫХ ЗАДАЧ ПРИ МАТЕМАТИЧЕСКОМ МОДЕЛИРОВАНИИ ГЕОЛОГИЧЕСКИХ СИСТЕМ
}

\author{
Тарасенко Елена Олеговна', \\ galail@mail.ru
}

\author{
Гладков Андрей Владимирович1, \\ gavandrew@mail.ru \\ 1 Северо-Кавказский федеральный университет, \\ Россия, 355009, г. Ставрополь, ул. Пушкина, 1, корпус 2.
}

\begin{abstract}
Актуальность. Математическое моделирование геологических систем является актуальным как теоретически, так и практически. Обратные прикладные задачи позволяют систематизировать знания об исследуемых моделях. В настоящей статье рассматриваются задачи об оценке глубины заложения заряда взрывчатого вещества в просадочных грунтах с целью их уплотнения и, как следствие, снижения просадочности. Такой метод уплотнения широко применяется при проведении работ на объектах гражданского строительства, изменении рельефрных особенностей местности и т. д. Следует отметить, что на территории России порядка 17 \% поверхности земной коры представлены просадочными лёссами.

Цель: аналитическое и численное моделирование решения обратных задач в геологических системах при уплотнении лёссов глубинными взрывами.
\end{abstract}

Методы: итерационные методы решения задач, методы компьютерного моделирования в рамках исследования геологических систем, статистика выводов.

Результаты. Предлагаются аналитические и численные решения рассматриваемой обратной прикладной задачи геологической системы. Описаны решения при условиях полного поглощения газа, образованного в результате взрыва сосредоточенного заряда, окружающим его просадочным грунтом (происходит уплотнение лёсса) и полного отражения газа от грунта (происходит выброс на поверхность). Подробно математически изучены различные состояния просадочного лёсса с характерными свойствами изотропности и анизотропности геологической системы. Проведён вычислительный эксперимент поиска приближённого итерационного решения обратной задачи для определения глубины проведения взрывов с целью уплотнения лёссов. Вычисления проводились с учётом имеющихся экспериментальных данных на примере объекта гражданского строительства Северного Кавказа.

Выводы. Проведённые исследования указывают на адекватность предлагаемого подхода оценки глубины заложения заряда. Численное решение задачи найдено с высокой точностью и с минимальными вычислительными затратами.

\section{Ключевые слова:}

Обратная задача, лёсс, уплотнение просадочных грунтов, математическое моделирование, численные методы.

\section{Введение}

Изучение геологических систем территории России показало, что порядка 17 \% поверхности земной коры представлены лёссами. Наибольшее распространение (не менее 80 \%) они получили в Северных регионах Кавказа, Южной части России, большей части территории Сибири (Западная, Восточная и Южная), Якутии, Поволжье и других регионах России.

Грунты Северного Кавказа представлены просадочными толщами второго типа грунтовых условий. В расположении лёссовых грунтов наблюдается 30нальность их залегания. При этом мощность просадочных слоёв составляет порядка 50-60 м, в отдельных районах до 100 м (Будённовский, Благодарненский районы Ставропольского края). Лёссы демонстрируют величину расчётной просадки до 2,5 м от собственного веса. Исследования геологических систем региона показали строение лёссовых грунтов с выраженным характером цикличности - расположение слоями в горизонтальной плоскости лёссовидных суглинков и лёссов с погребёнными почвами $[1,2]$.

В лёссах имеются в больших количествах макропоры, они позволяют воде глубоко проникать в структуру грунта, вследствие чего происходит его стремительное размокание и неравномерные деформации в виде просадок [3-7].
Для исключения просадочности лёссовых грунтов на площадках гражданского строительства или рельефных склонах территорий используется метод уплотнения грунтов глубинными взрывами [8-13]. В работах $[14,15]$ предложены математические модели геологических изменений при таком методе уплотнения. Авторы в [16] доказывают разрешимость краевых задач с заданными начальным и граничными условиями в рамках математического моделирования глубинного взрыва.

При проведении прикладного математического моделирования геологических систем возникает проблема решения и исследования разнообразных прямых и обратных задач [17]. В настоящем исследовании приведём решение обратной инженерно-геологической задачи - оценка глубины заложения взрывчатого вещества с целью уплотнения лёссов глубинными взрывами как аналитически, так и численно.

Математическое моделирование геологических систем при уплотнении лёссов глубинными взрывами

Математически диффузионный процесс проникновения атомов газа в процессе уплотнения лёссов глубинными взрывами в случае сосредоточенного заряда предлагается описать дифференциальным уравнением: 


$$
\begin{gathered}
\frac{\partial q}{\partial t}+u \frac{\partial q}{\partial x_{1}}+\varpi \frac{\partial q}{\partial x_{3}}+\alpha q= \\
=\frac{\partial}{\partial x_{1}} K_{x_{1}} \frac{\partial q}{\partial x_{1}}+\frac{\partial}{\partial x_{2}} K_{x_{2}} \frac{\partial q}{\partial x_{2}}+\frac{\partial}{\partial x_{3}} K_{x_{3}} \frac{\partial q}{\partial x_{3}}+f
\end{gathered}
$$

здесь $q$ - плотность уплотнённого грунта при глубинном взрыве; $u$ - параметр горизонтального распространения газа (вектор, направленный вдоль оси абсцисс $\left.O x_{1}\right) ; K_{x_{1}}, K_{x_{2}}, K_{x_{3}}$ - диффузионные парамет-

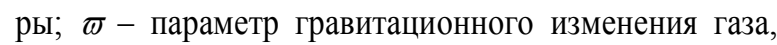
распространяемого в камуфлетной полости; $\alpha$ - параметр взаимодействия газа, появившегося в результате взрыва, и лёссового просадочного грунта; $f$ мощность заряда, описываемая функцией внешних сил воздействия на геологическую систему.

Учитывая, что в уравнении (1) параметры горизонтального распространения газа и диффузии определяются исходя из результатов измерений, уравнение диффузии является полуэмпирическим. Сравнивая (1) с известными видами дифференциальных уравнений, можно сделать вывод, что оно является линейным дифференциальным уравнениям в частных производных параболического типа.

Опишем диффузионное распространение атомов газа в результате глубинного грунтового взрыва сосредоточенным зарядом начально-граничной задачей [1411]

$$
\begin{gathered}
\frac{\partial q}{\partial t}+\sum_{i=1}^{3} u_{i} \frac{\partial q}{\partial x_{i}}+\alpha q+\sum_{i=1}^{3} \sum_{j=1}^{3} \frac{\partial}{\partial x_{i}} K_{i j} \frac{\partial q}{\partial x_{j}}=0, \\
q\left(t_{0}, x_{1}, x_{2}, x_{3}\right)= \\
=Q \cdot \delta\left(x_{1}-x_{1}^{0}\right) \delta\left(x_{2}-x_{2}^{0}\right) \delta\left(x_{3}-x_{3}^{0}\right), \\
t \in\left[t_{0}, T\right],\left.K_{33} \frac{\partial q}{\partial x_{3}}\right|_{x_{3}=x_{3}^{0}}=0, t>t_{0},
\end{gathered}
$$

при поверхностном выбросе, на глубине проведения взрыва $x_{3}$; начально-граничной задачей $(2),(3)$

$$
\left.q\left(t, x_{1}, x_{2}, x_{3}\right)\right|_{x_{3}=x_{3}^{0}}=0, t>t_{0},
$$

в условиях уплотнения лёсса; и краевой задачей (2), (3)

$$
\left.\left\{K_{33} \frac{\partial q}{\partial x_{3}}+\varpi q\right\}\right|_{x_{3}=x_{3}^{0}}=\left.\left\{v_{s} q\right\}\right|_{x_{3}=x_{3}^{0}},
$$

в условиях, когда атомы газа частично отражены и частично поглощены. В формуле (3) $Q$ задаёт мощность сосредоточенного заряда или количество выброшенного зарядом атомов газа в момент времени $t_{0}$ в точке $\left(x_{0}, y_{0}, z_{0}\right) ; \delta$ - единичная импульсная функция Дирака. В (6) $v_{s}$ - параметр, характеризующий величину общей скорости атомов газа в лёссе. Интенсивность потока газа, согласно равенству (6), реализуется через диффузионный поток

$$
\left.\left\{K_{33} \frac{\partial q}{\partial x_{3}}\right\}\right|_{x_{3}=x_{3}^{0}}
$$

и гравитационный поток

$$
\left.\{\varpi q\}\right|_{x_{3}=x_{3}^{0}} .
$$

Представленные граничные условия (4)-(6), описывающие математическую модель рассматриваемой геологической системы, показывают соответственно:

- лёсс полностью отражает газ,

- лёсс полностью поглощает газ,

- частичное отражение и частичное поглощение лёссом газа.

При поверхностном выбросе $z=0$ средние значения плотности и концентрации лёсса при реализации глубинного взрыва сосредоточенным зарядом определяются из выражения

$$
\left.\frac{\partial q(t, x, y, z)}{\partial z}\right|_{z=0}=0
$$

в условиях полного поглощения газа окружающим его грунтом (реализуется уплотнение лёсса) определяются из выражения

$$
q(t, x, y, 0)=0 .
$$

Аналитические представления решений соответствующих начально-граничных задач в рамках рассматриваемой геологической системы при реализации глубинного взрыва сосредоточенным снарядом описываются равенствами:

$$
\begin{gathered}
q_{1}(t, x, y, z)=\frac{Q}{(2 \pi)^{3 / 2} \sigma_{x} \sigma_{y} \sigma_{z} t^{3}} \times \\
\times \exp \left\{-\left(\frac{(x-\bar{U} t)^{2}}{2 \sigma_{x}^{2} t}+\frac{y^{2}}{2 \sigma_{y}^{2} t}\right)\right\} \times \\
\times\left[\exp \left\{-\frac{(z-H)^{2}}{2 \sigma_{z}^{2} t}\right\}+\exp \left\{-\frac{(z+H)^{2}}{2 \sigma_{z}^{2} t}\right\}\right], \\
q_{2}(t, x, y, z)=\frac{Q}{(2 \pi)^{3 / 2} \sigma_{x} \sigma_{y} \sigma_{z} t^{3}} \times \\
\times \exp \left\{-\left(\frac{(x-\bar{U} t)^{2}}{2 \sigma_{x}^{2} t}+\frac{y^{2}}{2 \sigma_{y}^{2} t}\right)\right\} \times \\
\times\left[\begin{array}{l}
\left.\exp \left\{-\frac{(z-H)^{2}}{2 \sigma_{z}^{2} t}\right\}-\exp \left\{-\frac{(z+H)^{2}}{2 \sigma_{z}^{2} t}\right\}\right],
\end{array}\right.
\end{gathered}
$$

где $\sigma_{x}^{2}(t), \sigma_{y}^{2}(t), \sigma_{z}^{2}(t)$ - дисперсионные координатные изменения атомов газа в момент времени $\mathrm{t}$ вдоль осей $\mathrm{Ox}, \mathrm{Oy}, \mathrm{Oz}$, соответственно, являются непрерывными функциями аргумента $t, t \geq 0$.

Равенства (7) и (8) применяются для построения решений отдельных обратных задач при проведении математического моделирования (2)-(4); (2), (3), (5), описывающие глубинный грунтовый взрыв сосредоточенными зарядами с целью уплотнения лёссов, исключения их просадочных свойств. 
Далее рассмотрим прикладные обратные задачи оценки глубины заложения сосредоточенных зарядов при математическом моделировании для снижения просадочных свойств лёссовых грунтов.

\section{Постановка обратных прикладных задач} геологических систем

Пусть известны средние значения плотности $q_{1}(t, x, y, z)$ уплотнённого грунта после проведения глубинного взрыва сосредоточенным зарядом в условиях поверхностного выброса, или пусть известны средние значения плотности $q_{2}(t, x, y, z)$ уплотнённого грунта после проведения глубинного взрыва сосредоточенным зарядом в условиях полного поглощения газа окружающим его грунтом (реализуется уплотнение лёсса), а также по заданной мощности сосредоточенного заряда или количества выброшенного зарядом атомов газа $Q$ и известным $\sigma_{x}^{2}(t), \sigma_{y}^{2}(t), \sigma_{z}^{2}(t)$ дисперсионным координатным изменениям атомов газа в момент времени $t$ вдоль осей $O x, O y, O z$ соответственно. Требуется оценить глубину заложения взрывчатого вещества $H$ с целью уплотнения лёссов глубинными сосредоточенными взрывами.

\section{Численное решение обратных задач}

Реализуем построение численных решений поставленных выше обратных прикладных задач итерационным методом [18], в рамках математического моделирования геологических систем.

1. Численный подход решения прикладной задачи (в условиях полного отражения атомов газа реализуется поверхностный выброс) опирается на применении итерационного метода. Если каким-либо способом (графически или аналитически) отделён отрезок $[a, b]$ ( $a$ и $b$ оценены, например, методом подбора), содержащий искомый корень $Н$ уравнения

$$
\begin{gathered}
q_{1}(t, x, y, z)-\frac{Q}{(2 \pi)^{3 / 2} \sigma_{x} \sigma_{y} \sigma_{z} t^{3}} \times \\
\times \exp \left\{-\left(\frac{(x-\bar{U} t)^{2}}{2 \sigma_{x}^{2} t}+\frac{y^{2}}{2 \sigma_{y}^{2} t}\right)\right\} \times \\
\times\left[\exp \left\{-\frac{(z-H)^{2}}{2 \sigma_{z}^{2} t}\right\}+\exp \left\{-\frac{(z+H)^{2}}{2 \sigma_{z}^{2} t}\right\}\right]=0,
\end{gathered}
$$

то поведение геологической системы с характерными свойствами анизотропности (в условиях полного отражения атомов газа) представим в виде

$$
H=\sigma_{z} \sqrt{-2 t \times \ln \left(\begin{array}{l}
\left.\left(\frac{q_{1}(t, x, y, z)(2 \pi)^{3 / 2} \sigma_{x} \sigma_{y} \sigma_{z} t^{3}}{Q}\right) \times\right) \\
\times \exp \left(\frac{(x-\bar{U} t)^{2}}{2 \sigma_{x}^{2} t}+\frac{y^{2}}{2 \sigma_{y}^{2} t}\right)- \\
-\exp \left\{-\frac{(z-H)^{2}}{2 \sigma_{z}^{2} t}\right\}
\end{array}\right)}-z .
$$

Итерационный процесс численного решения задачи оценки глубины заложения взрывчатого вещества $H$ построим по следующей формуле последовательных приближений:

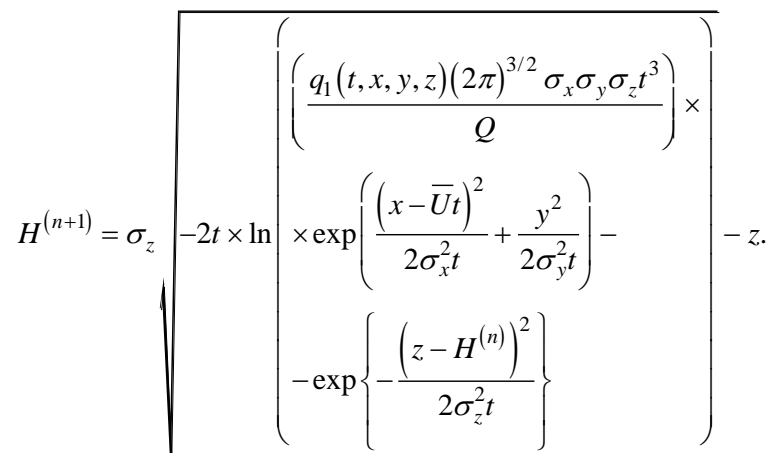

В предположении $\sigma_{z}=\sigma_{y}=\sigma_{x}$ поведение геологической системы с характерными свойствами изотропности $[1,19,20]$ (в условиях полного отражения атомов газа) представим в виде

$$
H=\sigma_{z} \sqrt{-2 t \times \ln \left(\begin{array}{l}
\left.\left(\frac{q_{1}(t, x, y, z)(2 \pi)^{3 / 2} \sigma_{z}^{3} t^{3}}{Q}\right) \times\right) \\
\times \exp \left(\frac{(x-\bar{U} t)^{2}+y^{2}}{2 \sigma_{z}^{2} t}\right)- \\
-\exp \left\{-\frac{(z-H)^{2}}{2 \sigma_{z}^{2} t}\right\}
\end{array}\right)}-z .
$$

Итерационный процесс численного решения задачи оценки глубины заложения взрывчатого вещества $H$ построим по следующей формуле последовательных приближений:

$$
H^{(n+1)}=\sigma_{z} \sqrt{-2 t \times \ln \left(\begin{array}{l}
\left(\frac{q_{1}(t, x, y, z)(2 \pi)^{3 / 2} \sigma_{z}^{3} t^{3}}{Q}\right) \times \exp \left(\frac{(x-\bar{U} t)^{2}+y^{2}}{2 \sigma_{z}^{2} t}\right)- \\
-\exp \left\{\frac{\left(z-H^{(n)}\right)^{2}}{2 \sigma_{z}^{2} t}\right\}
\end{array}\right)}-z .
$$

Построение последовательности приближений начнём с начального значения $H^{(1)}$ (первый шаг итераций), которое может принимать произвольное значение из отделённого отрезка $[a, b]$.

Критерием остановки поиска приближённого решения задачи итерационным методом служит достижение желаемой погрешности вычислений $\varepsilon$ и задаётся неравенством

$$
\left|H^{(n)}-H^{(n-1)}\right|<\varepsilon .
$$

2. Численный подход решения прикладной задачи (в условиях полного поглощения газа реализуется 
уплотнение лёсса) опирается на применении итерационного метода. Если каким-либо способом (графически или аналитически) отделён отрезок $[a, b]$ ( $a$ и $b$ оценены, например, методом подбора), содержащий искомый корень $H$ уравнения

$$
\begin{gathered}
q_{2}(t, x, y, z)-\frac{Q}{(2 \pi)^{3 / 2} \sigma_{x} \sigma_{y} \sigma_{z} t^{3}} \times \\
\times \exp \left\{-\left(\frac{(x-\bar{U} t)^{2}}{2 \sigma_{x}^{2} t}+\frac{y^{2}}{2 \sigma_{y}^{2} t}\right)\right\} \times \\
\times\left[\exp \left\{-\frac{(z-H)^{2}}{2 \sigma_{z}^{2} t}\right\}-\exp \left\{-\frac{(z+H)^{2}}{2 \sigma_{z}^{2} t}\right\}\right]=0,
\end{gathered}
$$

то поведение геологической системы с характерными свойствами анизотропности (в условиях полного поглощения газа) представим в виде

$$
H=\sigma_{z} \sqrt{-2 t \times \ln \left(\begin{array}{l}
\left.\left(\frac{q_{2}(t, x, y, z)(2 \pi)^{3 / 2} \sigma_{x} \sigma_{y} \sigma_{z} t^{3}}{Q}\right) \times\right) \\
\times \exp \left(\frac{(x-\bar{U} t)^{2}}{2 \sigma_{x}^{2} t}+\frac{y^{2}}{2 \sigma_{y}^{2} t}\right)+ \\
+\exp \left\{-\frac{(z-H)^{2}}{2 \sigma_{z}^{2} t}\right\}
\end{array}\right)-z .}
$$

Итерационный процесс численного решения задачи оценки глубины заложения взрывчатого вещества $H$ построим по следующей формуле последовательных приближений:

$$
H^{(n+1)}=\sigma_{z} \sqrt{-2 t \times \ln \left(\begin{array}{l}
\left(\frac{q_{2}(t, x, y, z)(2 \pi)^{3 / 2} \sigma_{x} \sigma_{y} \sigma_{z} t^{3}}{Q}\right) \times \exp \left(\frac{(x-\bar{U} t)^{2}}{2 \sigma_{x}^{2} t}+\frac{y^{2}}{2 \sigma_{y}^{2} t}\right)+ \\
+\exp \left\{-\frac{\left(z-H^{(n)}\right)^{2}}{2 \sigma_{z}^{2} t}\right\}
\end{array}\right)}-z .
$$

Поведение геологической системы с характерными свойствами изотропности $[1,19,20]$ (в условиях полного поглощения газа) в предположении $\sigma_{z}=\sigma_{y}=\sigma_{x}$, представим в виде

$$
H=\sigma_{z} \sqrt{-2 t \times \ln \left(\begin{array}{l}
\left(\frac{q_{2}(t, x, y, z)(2 \pi)^{3 / 2} \sigma_{z}^{3} t^{3}}{Q}\right) \times \exp \left(\frac{(x-\bar{U} t)^{2}+y^{2}}{2 \sigma_{z}^{2} t}\right)+ \\
+\exp \left\{-\frac{(z-H)^{2}}{2 \sigma_{z}^{2} t}\right\}
\end{array}\right)}-z .
$$

Итерационный процесс численного решения задачи оценки глубины заложения взрывчатого вещества $H$ построим по следующей формуле последовательных приближений:

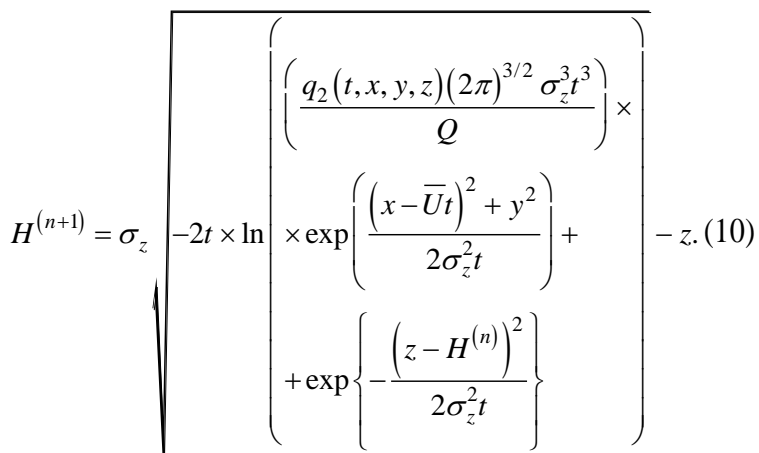

Построение последовательности приближений начнём с начального значения $H^{(1)}$ (первый шаг итераций), которое может принимать произвольное значение из отделённого отрезка $[a, b]$.

Критерием остановки поиска приближённого решения задачи итерационным методом служит достижение желаемой погрешности вычислений $\varepsilon$ и задаётся неравенством

$$
\left|H^{(n)}-H^{(n-1)}\right|<\varepsilon .
$$

\section{Вычислительный эксперимент}

Проведём вычислительной эксперимент для предлагаемых выше численных подходов решения обратных задач при математическом моделировании глубинного взрыва с целью исключения просадочности лёссов на объекте гражданского строительства.

Расчёты проведём с использованием экспериментальных данных, взятых из работы [1] и содержащих информацию о гидровзрывном уплотнении 20метровой просадочной толщи на объекте «Общежитие на 203 места Прикумского завода пластмасс в 7-м микрорайоне». Показатели плотности и влажности грунта после проведения глубинного взрыва сосредоточенным зарядом приведены в табл. $1 . \vec{u}=0 \mathrm{M} / \mathrm{c}-$ параметр горизонтального распространения газа (вектор, направленный вдоль оси абсцисс $O x$ ). При $\sigma_{z}=\sigma_{y}=\sigma_{x}$ геологическая система обладает характерными свойствами изотропности. Уплотнение реализуется в условиях полного поглощения газа окружающим его грунтом. Масса заряда 5 кг (всего использовано на площадке 136 зарядов, расположенных по сетке $4 \times 5$ м). Взрывы производились интервалом в 5 секунд.

Оценим глубину заложения взрывчатого вещества $H$ с точностью до $\varepsilon=0,001$.

Учитывая, что рассматриваемая геологическая система с характерными свойствами изотропности, решение задачи проведём по формуле (9). Дисперсионные координатные изменения атомов газа в момент времени $t$ вдоль оси $O z$ определим по плотности скелета грунта (табл. 1) $\sigma_{z} \in(1,61 ; 1,65)$.

Итерационный процесс численной оценки глубины заложения взрывчатого вещества $H$ вычислим по 
формуле последовательных приближений (10) с точностью до $\varepsilon=0,001$.

Таблица 1. Показатели плотности и влажности грунma [1]

Table 1. Indicators of soil density and humidity [1]

\begin{tabular}{|c|c|c|}
\hline $\begin{array}{c}\text { Глубина, м } \\
\text { Depth, m }\end{array}$ & $\begin{array}{c}\text { Влажность, \% } \\
\text { Humidity, \% }\end{array}$ & $\begin{array}{l}\text { Плотность скелета, } / \mathrm{cm}^{3} \\
\text { Skeleton density, } \mathrm{g} / \mathrm{cm}^{3}\end{array}$ \\
\hline 3,0 & 13,4 & 1,80 \\
\hline 4,0 & 14,2 & 1,77 \\
\hline 4,5 & 18,1 & 1,65 \\
\hline 5,0 & $13,8 \ldots 14,2$ & $1,62 \ldots 1,70$ \\
\hline 6,0 & $12,1 \ldots 14,1$ & $1,61 \ldots 1,65$ \\
\hline 7,4 & $14,8 \ldots 15,0$ & $1,61 \ldots 1,65$ \\
\hline 8,7 & $17,3 \ldots 17,4$ & $1,61 \ldots 1,62$ \\
\hline 9,1 & $15,7 \ldots 17,0$ & $1,63 \ldots 1,75$ \\
\hline 10,0 & 17,4 & 1,75 \\
\hline 11,0 & $15,3 \ldots 16,5$ & $1,61 \ldots 1,68$ \\
\hline 11,3 & 14,7 & 1,64 \\
\hline
\end{tabular}

В табл. 2 представлены экспериментальные и вычисленные значения оценки глубины заложения сосредоточенного заряда в просадочный грунт.

Таблица 2. Экспериментальные и вычисленные значения оценки глубины заложения взрывчатого вещества $H$, м, на каждом шаге итерации

Table 2. Experimental and calculated values for estimating the depth of the explosive $H, m$, at each iteration step

\begin{tabular}{|c|c|c|}
\hline \multirow{2}{*}{$\begin{array}{l}\text { Номер итерации } \\
\text { Iteration number }\end{array}$} & \multicolumn{2}{|c|}{$H, \mathrm{~m} / \mathrm{m}$} \\
\hline & $\begin{array}{c}\text { экспериментальная } \\
\text { experimental }\end{array}$ & $\begin{array}{l}\text { вычисленная } \\
\text { calculated }\end{array}$ \\
\hline 1 & \multirow{10}{*}{6} & 6,1307 \\
\hline 2 & & 6,0743 \\
\hline 3 & & 6,0524 \\
\hline 4 & & 6,0408 \\
\hline 5 & & 6,0296 \\
\hline 6 & & 6,0218 \\
\hline 7 & & 6,0172 \\
\hline 8 & & 6,0053 \\
\hline 9 & & 6,0018 \\
\hline 10 & & 6,0016 \\
\hline
\end{tabular}

Критерием остановки поиска приближённого решения задачи итерационным методом послужило достижение желаемой погрешности вычислений $\varepsilon$

$$
\left|H^{(10)}-H^{(9)}\right|<0,001 .
$$

На рисунке представлены графические реализации экспериментальных и вычисленных значений оценки глубины заложения сосредоточенного заряда $H$ на каждом шаге построения последовательности итераций.

Проведённый вычислительный эксперимент продемонстрировал с точностью до $\varepsilon=0,001$ адекватность численных расчётов экспериментальным данным.

\section{СПИСОК ЛИТЕРАТУРЬ}

1. Галай Б.Ф. Уплотнение просадочных грунтов глубинными взрывами. - Ставрополь: Сервисшкола; СКФУ, 2015. - 240 с.

2. Галай Б.Ф. Пособие по уплотнению просадочных лессовых грунтов глубинными взрывами в условиях Северного Кавказа (изыскания, проектирование, производство работ)/ изд. 3-е, доп. - Ставрополь: Сервисшкола; СКФУ, 2016. - 142 с.
Была получена оценка глубины заложения сосредоточенного заряда взрывчатого вещества $H=6,0018 \approx 6$ м за девять итераций.

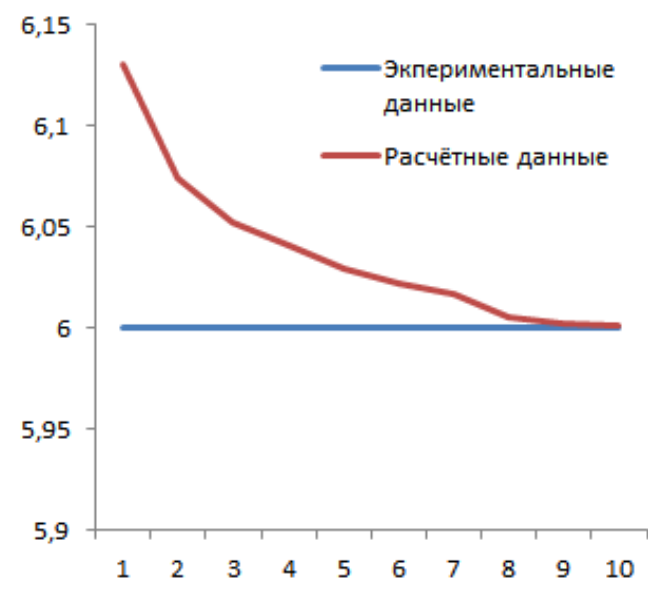

Рисунок. Экспериментальные (прямая) и вычисленные (ломанная) значения оченки глубины заложения сосредоточенного заряда Н на каждом шаге uтерации $n$

Figure. Experimental (straight line) and calculated (broken) values of the estimate of the depth of the concentrated charge $H$ at each iteration step $n$

\section{Заключение}

Проведение математического моделирования уплотнения просадочных грунтов глубинными взрывами неизбежно приводит к обратным прикладным задачам, решению которых посвящено настоящее исследование. В работе приведено построение численных решений обратных задач - оценка глубины заложения взрывчатого вещества в геологических системах с характерными свойствами анизотропности и изотропности.

Проведённый вычислительный эксперимент демонстрирует адекватность предлагаемого подхода оценки глубины заложения взрывчатого сосредоточенного заряда натурному уплотнению лёсса на объекте гражданского строительства. Получено численное решение обратной прикладной задачи с точностью до $\varepsilon=0,001$.

При проведении вычислительного эксперимента построенный итерационный процесс демонстрировал сходимость и приводил к решению задачи с различными начальным приближением и точностью. При этом наблюдалось существенное изменение вычислительной сложности решаемой задачи (количество итераций).

Авторы выражают признательность профессору Борису Фёдоровичу Галаю за оказанную помощь при проведении данного научного исследования.

3. Свидерских А.В., Носков И.В. Анализ изменения характеристик лессовых грунтов в зависимости от пористости // Вестник Евразийской науки, 2019. - Т. 11. - № 2. URL: https://esj.today/ PDF/76SAVN219.pdf (дата обращения 15.11.2021).

4. Ivanov P.L. Compaction of cohesionless soils by explosives // Proc. of the VI International Conference on Soil Mechanics and Foundation Engineering. - Monreal, 1966. - V. 3. - P. 352-354. 
5. Amŝiejus J., Dirgèlienė N. Probabilistic assessment of soil shear strength parameters using triaxial test result // The Baltic Journal of Road and Bridge Engineering. - 2007. - V. 2. - № 3. - P. 125-131.

6. Интерпретация прочностных характеристик грунта для численных исследований / А.А. Петраков, А.Ю. Прокопов, Н.А. Петракова, М.Д. Панасюк // Известия Тульского государственного университета. Науки о Земле. - 2021. - № 1. C. $225-236$.

7. Пантюшина Е.В. Лёссовые грунты и инженерные методы устранения их просадочных свойств // Ползуновский вестник. - 2011. - № 1. - С. 127-130.

8. Hall C.E. Compacting a dam foundation by blasting // Journal of Soil Mechanics and Foundation Division. - 1962. - V. 88. № SM3. - P. 31-51.

9. Liman A.K. Compaction of cohesionless foundation soils by explosives// Civil Engineering. - 1940. - V. 10. - Iss. 4. - P. 9-15.

10. Consolidation of organic subsoil's by applying underground explosions for formations of vertical sands drains and generating dynamic overloading / E. Dembicki, N. Kisielowa, R. Bona, R. Imiolek, A. Michowski, I. Semrau // Proc. 9 Int. Harbour Congress. - Antwerp, 1988. - P. 5.165-5.170.

11. Dembicki E. Dynamic consolidation of organic subsoil's by use of hidden underground explosions / E. Dembicki, N. Kisielowa, R. Bona, R. Imiolek, A. Michowski, I. Semrau // Proc. of the II Baltic Conf. S. M. F. E. - Tallinn, 1988. - P. 273-277.

12. Гридневский А.В., Прокопов А.Ю. Природно-техногенные условия формирования подтопления межбалочных пространств города Ростова-на-Дону // Известия Тульского государственного университета. Науки о Земле. - 2019. - № 2. C. 26-37.

13. Способ уплотнения лёссовых грунтов в основании зданий и сооружений: пат. Рос. Федерация, № 2015247, заявл. 27.12.1991; опубл. 30.06.1994. Бюл. № 46. - 6 с.
14. Тарасенко Е.О., Тарасенко В.С., Гладков А.В. Математическое моделирование уплотнения просадочных лёссовых грунтов Северного Кавказа глубинными взрывами // Известия Томского политехнического университета. - 2019. - Т. 330. № 11. - C. 94-101. DOI: 10.18799/24131830/2019/11/2352.

15. Математическое моделирование сосредоточенного глубинного взрыва на основе уравнения диффузии / Е.О. Тарасенко, А.В. Гладков, П.К. Корнеев, В.С. Тарасенко // Естественные науки - основа настоящего и фундамент для будущего: Материалы VI ежегодной научно-практической конференции Северо-Кавказского федерального университета «Университетская наука - региону». - Ставрополь: Изд-во СевероКавказского Федерального университета, 2018. - С. 66-68.

16. Тарасенко Е.О., Гладков А.В., Маликова Н.В. Разрешимость краевых задач, описывающих диффузию атомов пленки в подстилающей поверхности при образовании тонкопленочных структур // Известия Томского политехнического университета. Инжиниринг георесурсов. - 2016. - Т. 327. - № 2. C. $125-132$

17. Денисов А.М. Введение в теорию обратных задач. - М.: Издво Московского государственного университета, 1994. - 208 с.

18. Вержбицкий В.М. Численные методы: (математический анализ и обыкновенные дифференциальные уравнения). - М.: Директ-Медиа, 2013. - 400 с.

19. Tsukamoto Y., Ishihara K. Analysis on settlement of soil deposits following liquefaction during earthquakes // Soils and Foundation. - 2010. - № 50 (3). - P. 399-441.

20. Ishihara K. New challenges in Geotechnique for ground hazards due to intensely strong earthquake shaking // Geotechnical, Geological and Earthquake Engineering. - 2009. - № 11. - P. 91-114.

Поступила 01.12.2021 г.

\section{Информация об авторах}

Tapaceнко E.O., кандидат физико-математических наук, доцент, доцент кафедры вычислительной математики и кибернетики Факультета математики и компьютерных наук имени профессора Н.И. Червякова СевероКавказского федерального университета.

Гладков A.B., старший преподаватель кафедры вычислительной математики и кибернетики Факультета математики и компьютерных наук имени профессора Н.И. Червякова Северо-Кавказского федерального университета. 
UDC 51-74

\title{
NUMERICAL SOLUTION OF INVERSE PROBLEMS IN MATHEMATICAL MODELING OF GEOLOGICAL SYSTEMS
}

\author{
Elena 0. Tarasenko', \\ galail@mail.ru
}

\author{
Andrey V. Gladkov ${ }^{1}$, \\ gavandrew@mail.ru \\ 1 North-Caucasian Federal university, \\ 1, bld. 2, Pushkin street, Stavropol, 355009, Russia.
}

The relevance of the research. Mathematical modeling of geological systems is relevant both theoretically and practically. Inverse applied problems make it possible to systematize knowledge about the models under study. This article deals with the problem of assessing the depth of an explosive charge in collapsible soils in order to compact them and, as a consequence, reduce subsidence. This method of compaction is widely used at civil construction sites, relief features of the territories, etc. It should be noted as well that Russian territories are presented by loesses in about $17 \%$ of cases.

The main aim of the research is analytical and numerical modeling of the solution of inverse problems in geological systems when compacting loesses by deep explosions.

Methods: iterative methods for solving problems, methods of computer modeling in the study of geological systems, conclusion statistics. Results. Analytical and numerical solutions of the considered inverse applied problem of the geological system are proposed. Solutions are described under the conditions of complete absorption of the gas formed as a result of the explosion of a concentrated charge by the surrounding collapsing soil (the loess is compacted) and the complete reflection of the gas from the soil (the release to the surface occurs). Various states of subsidence loess with characteristic properties of isotropy and anisotropy of the geological system have been studied in detail mathematically. A computational experiment was carried out to find an approximate iterative solution to the inverse problem for determining the depth of explosions in order to compact loess. The calculations were carried out taking into account the available experimental data on the example of a civil construction object in the North Caucasus.

Conclusions. The studies carried out indicate the adequacy of the proposed approach for assessing the depth of the charge. The numerical solution of the problem is found with high accuracy and with minimal computational costs.

\section{Key words:}

Inverse problem, loess, compaction of subsident soils, mathematical modeling, numerical methods.

The authors express their gratitude to Professor Boris Fedorovich Galay for his assistance in conducting this scientific research.

\section{REFERENCES}

1. Galay B.F. Uplotnenie prosadochnykh gruntov glubinnymi vzryvami [Compaction of subsidence soils by deep explosions]. Stavropol, Servisshkola Publ., NCFU, 2015. 240 p.

2. Galay B.F. Posobie po uplotneniyu prosadochnykh lessovykh gruntov glubinnymi vzryvami $v$ usloviyakh Severnogo Kavkaza (izyskaniya, proektirovanie, proizvodstvo rabot) [Manual on compaction of subsidence loess soils by deep explosions in the conditions of the North Caucasus (research, design, production of works)]. $3^{\text {rd }}$ ed., add. Stavropol, Servisshkola Publ., NCFU, 2016. 142 p.

3. Sviderskikh A.V., Noskov I.V. Analysis of characteristics changes in the loess soils depending on porosity. The Eurasian Scientific Journal, 2019, vol. 11, no. 2. In Rus. Available at: https://esj. today/PDF/76SAVN219.pdf (accessed 15 November 2021).

4. Ivanov P.L. Compaction of cohesionless soils by explosives. Proc. of the VI International Conference on Soil Mechanics and Foundation Engineering. Monreal, 1966. Vol. 3, pp. 352-354.

5. Amŝiejus J., Dirgèlienè N. Probabilistic assessment of soil shear strength parameters using triaxial test result. The Baltic Journal of Road and Bridge Engineering, 2007, vol. 2, no. 3, pp. 125-131.

6. Petrakov A.A., Prokopov A.Yu., Petrakova N.A., Panasyuk M.D Interpretation of soil strength characteristics for numerical studies. Bulletin of the Tula State University. Science about Earth, 2021, no. 1, pp. 225-236. In Rus.

7. Pantyushina E.V. Loess soils and engineering methods for eliminating their subsidence properties. Polzunovsky vestnik, 2011, no. 1, pp. 127-130. In Rus.

8. Hall C.E. Compacting a dam foundation by blasting. Journal of Soil Mechanics and Foundation Division, 1962, vol. 88, no. SM3, pp. 31-51.
9. Liman A.K. Compaction of cohesionless foundation soils by explosives. Civil Engineering, 1940, vol. 10, Iss. 4, pp. 9-15.

10. Dembicki E., Kisielowa N., Bona R., Imiolek R., Michowski A., Semrau I. Consolidation of organic subsoil's by applying underground explosions for formations of vertical sands drains and generating dynamic overloading. Proc. 9 Int. Harbour Congress. Antwerp, 1988. pp. 5.165-5.170.

11. Dembicki E., Kisielowa N., Bona R., Imiolek R., Michowski A., Semrau I. Dynamic consolidation of organic subsoil's by use of hidden underground explosions. Proc. of the II Baltic Conf. S. M. F. E. Tallinn, 1988. pp. 273-277.

12. Gridnevsky A.V., Prokopov A.Yu. Natural and technogenic conditions of the formation of flooding of the inter-girder spaces of the city of Rostov-on-Don. Proceedings of the Tula State University. Earth Sciences, 2019, no. 2, pp. 26-37. In Rus.

13. Osipov V.I., Filimonov S.D., Melnikov B.N., Kail E.V. Sposob uplotneniya lyossovykh gruntov $v$ osnovanii zdaniy $i$ sooruzheniy [Method of compaction of loess soils in the base of buildings and structures]. Patent RF, no. 2015247, 1994.

14. Tarasenko E.O., Tarasenko V.S., Gladkov A.V. Mathematical modeling of consolidation of subsidence loess soils of the North Caucasus by deep explosions. Bulletin of the Tomsk Polytechnic University. Geo Assets Engineering, 2019, vol. 330, no. 11, pp. 94-101. In Rus. DOI: 10.18799/24131830/2019/11/2352.

15. Tarasenko E.O., Gladkov A.V., Korneev P. K., Tarasenko V. S. Matematicheskoe modelirovanie sosredotochennogo glubinnogo vzryva na osnove uravneniya diffuzii [Mathematical modeling of a concentrated deep explosion based on the diffusion equation]. Estestvennye nauki - osnova nastoyashchego i fundament dlya budushchego. Materialy VI ezhegodnoy nauchno-prakticheskoy konferentsii Severo-Kavkazskogo federalnogo universiteta «Universi- 
tetskaya nauka - regionu» [Natural sciences - the basis of the present and the foundation for the future. Materials of the VI Annua scientific and practical Conference of the North Caucasus Federal University «University Science - to the region»]. Stavropol, North Caucasus Federal University Publ. house, 2018. pp. 66-68.

16. Tarasenko E.O., Gladkov A.V., Malikova N.V. Solvability of boundary value problems describing the diffusion of film atoms in the underlying surface during the formation of thin-film structures. Bulletin of the Tomsk Polytechnic University. Geo Assets Engineering, 2016, vol. 327, no. 11, pp. 125-132. In Rus.

17. Denisov A.M. Vvedenie v teoriyu obratnykh zadach [Introduction to the theory of inverse problems]. Moscow, Moscow State University Press, 1994. 208 p.
18. Verzhbitsky V.M. Chislennye metody: (matematicheskiy analiz $i$ obyknovennye differentsialnye uravneniya) [Numerical methods: (mathematical analysis and ordinary differential equations)]. Moscow, Direct-Media Publ., 2013. 400 p.

19. Tsukamoto Y., Ishihara K. Analysis on settlement of soil deposits following liquefaction during earthquakes. Soils and Foundation, 2010, no. 50 (3), pp. 399-441.

20. Ishihara K. New challenges in Geotechnique for ground hazards due to intensely strong earthquake shaking. Geotechnical, Geological and Earthquake Engineering, 2009, no. 11, pp. 91-114.

Received: 1 December 2021.

\section{Information about the authors}

Elena O. Tarasenko, Cand. Sc., assistant professor, North-Caucasian Federal University.

Andrey V. Gladkov, senior teacher, North-Caucasian Federal University. 